\title{
Determinação do número pi $(\pi)$ por meio de uma rede quadrada de resistores idênticos
}

\author{
Determination of the pi $(\pi)$ number through square net of identical resistors
}

\author{
E.V.C. Tadeu ${ }^{1}$, L. M. Alves ${ }^{1}$, M. I. A. Jardim ${ }^{1}$, W. P. Queirós*1, A. M. B. Goncalves ${ }^{1}$ \\ ${ }^{1}$ Instituto de Física, Universidade Federal do Mato Grosso do Sul, Campo Grande, MS, Brasil.
}

Recebido em 27 de Abril, 2017. Revisado em 27 de Julho, 2017. Aceito em 28 de Julho, 2017.

\begin{abstract}
O número $\pi$ em geral é descrito como o valor obtido da razão perímetro por diâmetro de uma circunferência, mas esse número aparece em várias circunstâncias nas ciências em geral, principalmente quando um sistema possui periodicidade. Neste trabalho apresentaremos uma forma de se obter experimentalmente essa constante matemática através da medida da resistência equivalente em uma malha infinita quadrada de resistores. As medidas foram realizadas ao longo do processo de construção da malha usando resistores reais e simulando-os em um aplicativo online. As medidas mostram que quanto maior a quantidade de camadas da malha, mais próximo do valor de $\pi$ se aproxima. Apresentaremos também, outras as formas alternativas de realizar o experimento.

Palavras-chave: Medida de $\pi$, Malha infinita de resistores.
\end{abstract}

The number $\pi$ is generally described as the value obtained from the perimeter per diameter ratio of a circumference. But this number appears in various circumstances in the sciences in general, especially when a system has periodicity. In this work we present a way to obtain this mathematical constant experimentally by measuring the equivalent resistance in an resistors infinite square grid. Measurements were made throughout the fabrication process using real resistors and simulating them in an online application. The measurements show that the greater the number of layers of the mesh, the closer the value of $\pi$ is reached. We will also present other alternative ways of performing such an experiment.

Keywords: Measurement of $\pi$, infinity resistors web.

\section{Introdução}

Desde 4000 anos, aproximadamente, sabe-se que a razão entre o Comprimento e o diâmetro de uma circunferência é constante $[1,2]$. Modernamente, chamamos esta constante fundamental de "pi", representada pela letra grega $(\pi)$ e expressa por um número irracional, cujo o valor é aproximadamente 3,14 . Os primeiros estudos para determinar o valor de $\pi$, encontram-se no Papiro de Rhind escrito, aproximadamente, em 1700 a.C., no Egito Antigo [1]. Rhind procedeu da seguinte forma: A área de um círculo é igual à de um quadrado, cujo lado é o diâmetro do círculo diminuído de sua nona parte:

$$
\pi R^{2}=\left(2 R-\frac{2 R}{9}\right)^{2} \rightarrow \pi=\frac{16^{2}}{9^{2}}=\frac{256}{81} \approx 3,1604 \pi R^{2}
$$

Posteriormente, ao logo da história, encontramos outras referências ao valor do número $\pi$. Os babilônios mostraram que o valor de $\pi$ situa-se entre $3_{8}^{1} e 3{ }_{7}^{1}$, ou seja, $\frac{25}{8}<\pi<\frac{22}{7}$. O que em frações decimais, equivale a $3,125<\pi<3,142$. Os escritos do velho testamento, há cerca de 500 anos a. C., possuem um trecho em que declara o valor de $\pi$ é igual a 3 [2]. Arquimedes em,

*Endereço de correspondência: wellington_fis@yahoo.com.br. aproximadamente, 200 a. C., em seu trabalho intitulado "Sobre a medida do círculo" usou um método árduo e partindo de um hexágono regular, calculou os perímetros e áreas de hexágonos de 6, 12, 24, 48 e 96 lados inscritos e circunscritos em uma circunferência e mostrou que $\pi$ estava entre 3,14084 e 3,14285. O método de Arquimedes foi considerado o melhor para o cálculo de $\pi$ até o advento do cálculo diferencial e integral [1]. Bongiovanni e Watanabe [1] citam alguns nomes e datas do processo da universalidade de cálculo de $\pi$ em diversos períodos:

Ainda na Grécia antiga Ptolomeu (87-165) calculou as cordas de todos os ângulos de meio e meio grau, entre 0 e 180 graus, e obteve o valor aproximado de 3,14166. Na índia, Aryabhata $(\approx 500)$ e, posteriormente, Bhaskara $(\approx 1140)$ obtiveram para $\pi$ o valor $3927 / 1250 \approx 3,1416$, calculando os perímetros de polígonos de 12, 24, 48, 96 e 384 lados. Para Brahmagupta $(\approx 628), \pi=\sqrt{10} . \mathrm{Na}$ China, no século 12 a. C.; o valor de $\pi$ era 3 e, no início da era cristã, $\sqrt{10}$. Tsu Ch'ungchih (430-501) obteve para $\pi$ o valor $355 / 113$ $(\approx 3,1415929)$, valor este redescoberto na Europa 1000 anos depois. Nos séculos 15 e 16, 
com o desenvolvimento da trigonometria e uma notação melhor para números, a determinação dos comprimentos de cordas tornouse mais precisa e mais rápida. Matemáticos dessa época, ainda usando o método de Arquimedes, calcularam $\pi$ com até 35 casas decimais $[1$, p. 3$]$.

Com o advento do cálculo, no século XVII, a determinação de $\pi$ passa por uma nova fase com novas ferramentas que substituem os métodos geométricos. Exemplo disso, temos os cálculos feitos por meio de uma série publicada independentemente por Gregory, em 1670, e publicado por Leibniz em 1674: $1-1 / 3+1 / 5-1 / 7+\ldots=\quad \frac{\pi}{4}$. Esta série, por convergir muito lentamente, não é muito conveniente para o cálculo de $\pi$, no entanto, possui um valor estético especial $[1,2]$. Depois do cálculo feito por Gregory, por meio de algumas relações trigonométricas, mais casas decimais de $\pi$ foram determinadas em tempos cada vez menores.

Quanto à natureza do número $\pi$, desde que os estudos sobre número irracional tornaram-se consolidados, houve indícios de que o número $\pi$ pudesse ser irracional. Johann Heinrich Lambert (1728-1777), em 1761, demonstrou que $\pi$ é um número irracional. Mais tarde em 1947 uma demonstração simplificada foi feita por Ivan Morton Niven (1917-1999). Euler conjecturou que $\pi$ seria um número transcendente, ou seja, não poderia ser raiz de um polinômio com coeficientes inteiros. Depois da morte de Euler, em 1882, Carl Louis Ferdinand Von Lindemann (1852-1939) provou que $\pi$ é transcendente $[1,2]$.

$\mathrm{Na}$ era da informática, a quantidade de casa decimais na determinação de $\pi$ aumentou consideravelmente. O primeiro cálculo de $\pi$ pelo computador, foi realizado em 1949, com 2036 casas decimais. Em setembro de 1989 a revista Science News noticia que David e Gregory Chudnoviski calcularam $\pi$ com 1 bilhão de algarismos decimais exatos [1]. Recentemente, em 2002, Kanada et al. calcularam $\pi$ com 1.241.100.000.000 de casas decimais [2]. A principal finalidade de cálculos cada vez mais precisos do $\pi$ é a demonstração da potência de supercomputadores, bem como, novos algoritmos e métodos de cálculo.

A importância de $\pi$ dá-se também por sua relação em estudos de várias ciências, como exemplo: nas áreas biológicas, descrevendo a hélice dupla do DNA; na arquitetura e na economia para entender o padrão do mercado de capitais; na Física, na teoria das supercordas, nas equações de Einstein do campo gravitacional, na teoria das vibrações e movimentos ondulatórios. Uma das possibilidades de cálculo experimental de $\pi$ mais comum é o estudo do movimento de um pêndulo simples. Sabemos que o período de um pêndulo simples, quando balançado com uma pequena amplitude, pode ser calculado pela equação: $T \approx 2 \pi \sqrt{L / g}$ onde $L$ é o comprimento do fio e $g$ a aceleração da gravidade no local do experimento. As grandezas da equação são facilmente medidas em laboratório, o que permite estimar o valor de $\pi$.
Nesse sentido, o presente trabalho apresentará mais um exemplo no campo da Física, de como se obter o valor de $\pi$, por meio da medida da resistência entre dois pontos adjacentes de uma rede quadrada infinita de resistores idênticos de resistência $R$. Isto será possível porque todos os sistemas que apresentam periodicidade (cíclicos) apresentam o valor de $\pi$ em algum momento.

A proposta apresentada no presente estudo pode ser utilizada no Ensino de Física, em nível de Ensino Médio e nas disciplinas de Laboratório de Física dos cursos de graduação. A atividade mostra-se importante, pois pode contribuir para o favorecimento da interdisciplinaridade, uma vez que aborda a relação entre os conteúdos de Física e Matemática.

\subsection{A malha infinita de resistores}

Em física, existe um problema muito interessante que é o cálculo da resistência equivalente em malhas infinitas de resistores. Estas podem ser desde as mais simples (unidimensionais, ou escada infinita) como mais complexos sendo bi ou tridimensionais. Neste trabalho, tomamos como ponto de partida o seguinte problema: "Qual é a resistência entre os pontos A e B de uma malha infinita de resistores como esquematizado na figura 1?"

A solução desse problema já foi demonstrada em diversos artigos [3] e envolve o uso das funções de rede de Green. A solução parte do princípio de que a malha infinita é um sistema periódico. Considerando resistores de resistência $R$, o valor da resistência equivalente $R_{\text {eq }}$ calculado entre os pontos A e B Figura 1, deve ser:

$$
R_{e q}=\frac{2 R}{\pi}
$$

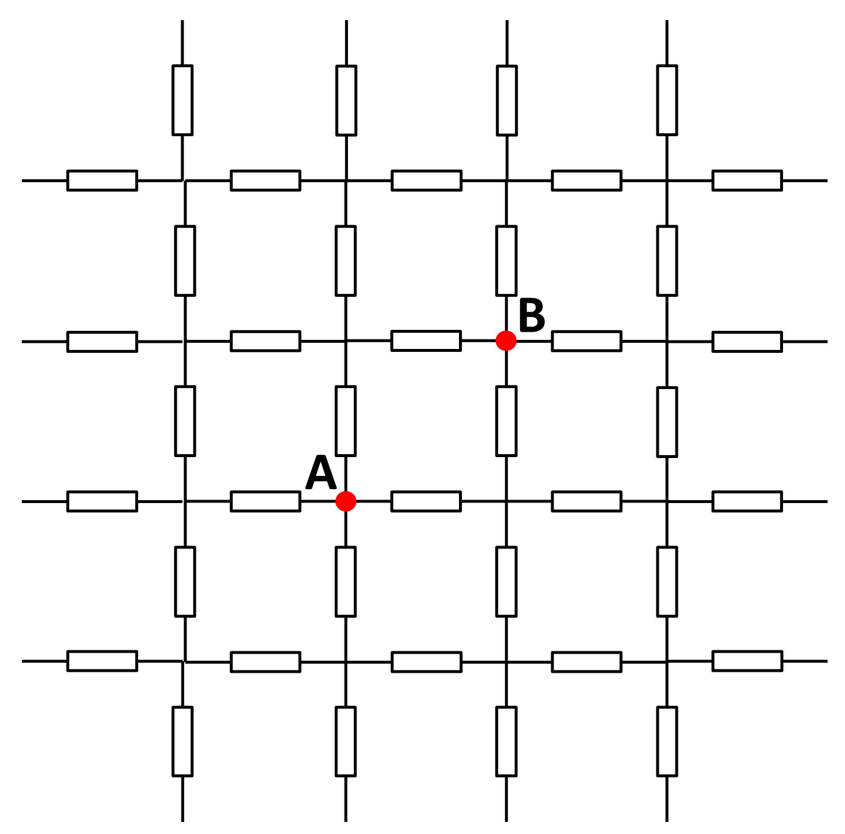

Figura 1: Representação da malha infinita de resistores e os dois pontos (em vermelho) que interessam. 
Com base nesse problema vê-se que é possível medir a constante $\pi$ construindo uma malha de resistores, grande o suficiente, e medindo a resistência com o auxílio de um multímetro. Neste trabalho apresentamos o resultado da medida experimental da resistência na diagonal dada pelos pontos A e B da figura 1 de uma malha infinita de resistores e comparação com o resultado de uma simulação computacional da mesma.

\section{Metodologia}

Para medir experimentalmente a constante $\pi$ foi construída uma malha quadrada de resistores soldando-os um a um Figura 2(a). Para cada camada terminada, medimos o valor da resistência equivalente nos dois pontos marcados utilizando um multímetro de alta precisão. A camada 0 é definida pelos quatro resistores Figura 2(b) que formam o quadrado central e cujos pontos que formam sua diagonal foi medida a resistência equivalente. Ao todo foram usados 480 resistores de $(560 \pm 20) \Omega$. O objetivo de medir o sistema camada a camada foi para chegar a um número de camadas mínimo (ou suficiente) para garantir uma boa aproximação, uma vez que a teoria considera uma malha infinita de resistores e experimentalmente isso não é possível. No final foram construídas 7 camadas. $\mathrm{O}$ valor de $\pi$ foi calculado então a partir da resistência equivalente medida nos dois pontos e da resistência nominal dos resistores $(\mathrm{R})$ utilizados como mostrado abaixo:

$$
\pi=\frac{1120}{R_{e q}}
$$

onde $R_{\text {eq }}$ é a resistência equivalente medida.

Como forma de comparar nossos resultados fizemos uma simulação do circuito de resistores utilizando o aplicativo online 123D Circuits da Autodesk [4]. Neste simulador de circuitos realizamos a montagem e medidas na mesma metodologia do circuito real, ou seja, medimos a resistência equivalente do circuito a cada nova camada construída. Também, para esta simulação, usamos os

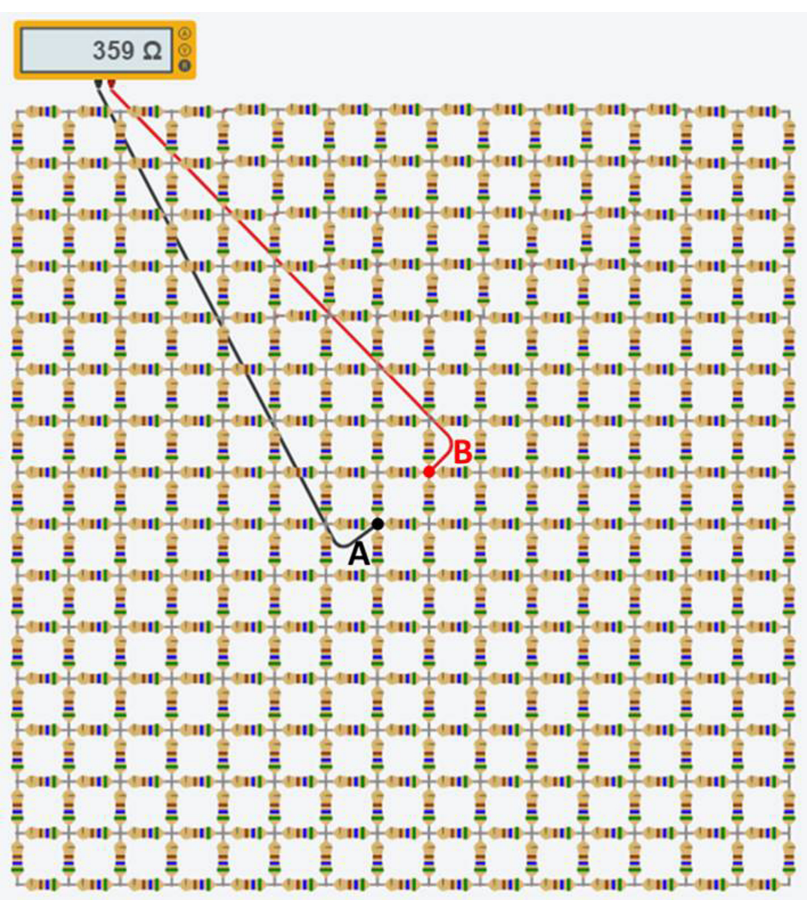

Figura 3: Rede de resistores montada no aplicativo [4]. Pode-se ver também no canto superior esquerdo um ohmímetro e os fios ligando ele aos pontos A e B. O valor apresentado é o valor da resistência equivalente, entre os pontos, medida na simulação.

resistores no mesmo valor dos usados na montagem experimental $(560 \Omega)$. A Figura 3 mostra o circuito que foi construído no simulador.

\section{Resultados e Discussão}

As tabelas 1 e 2 apresentam os resultados medidos para a resistência equivalente medida (experimento e simulação respectivamente) e o valor de $\pi$ calculado com base na equação 2 após cada camada construída. Também é apresentado o valor de $\Delta \pi$ que representa o ganho no
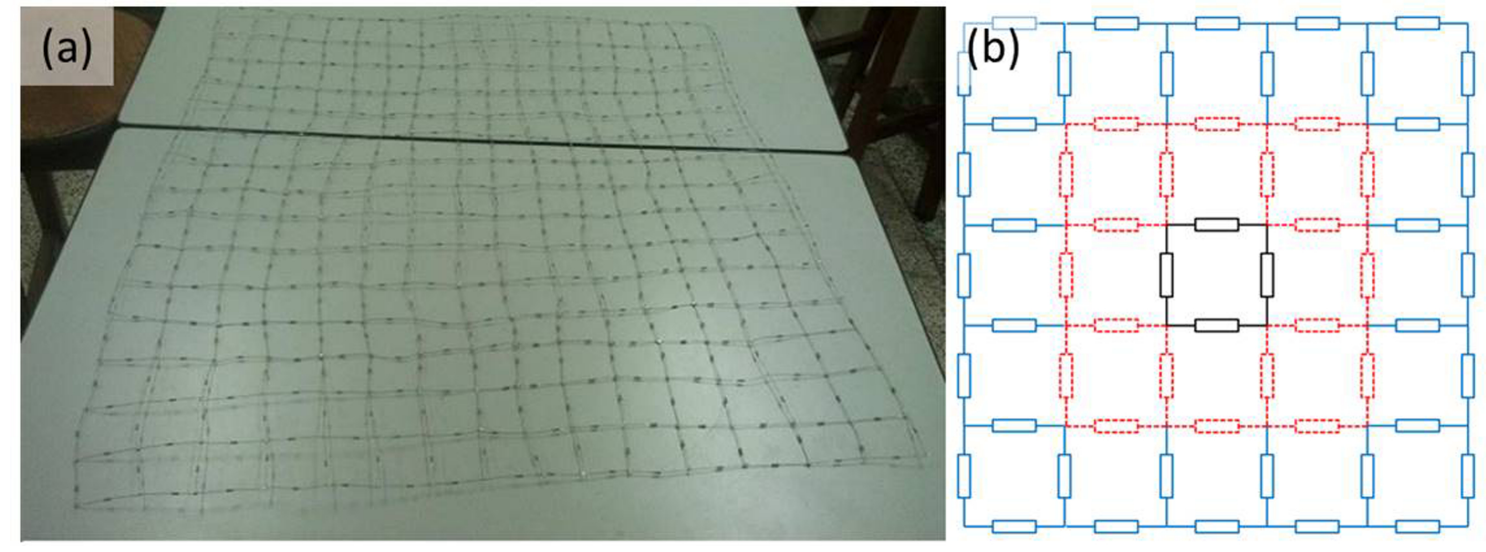

Figura 2: (a) Rede de resistores construída com 7 camadas. (b) Esquema de como são definidas as camadas: A camada 0 é definida pelos quatro resistores em preto que formam o quadrado central, a camada 1 é definida pelos resistores em vermelho tracejado que rodeiam a camada 0 e a camada 2 é definida pelos resistores azuis. 
Tabela 1: Resultados experimentais e cálculos para a rede quadrada de resistores em função do número de camadas.

\begin{tabular}{lccc}
\hline N. de camadas & $\mathrm{R}_{\text {eq }}(\mathrm{k} \Omega)$ & Cálculo de $\pi$ & $\Delta \pi$ \\
\hline 1 & 397,88 & 2,815 & - \\
\hline 2 & 383,28 & 2,922 & 0,107 \\
\hline 3 & 366,91 & 3,053 & 0,130 \\
\hline 4 & 361,62 & 3,097 & 0,045 \\
\hline 5 & 359,23 & 3,118 & 0,021 \\
\hline 6 & 358,18 & 3,127 & 0,009 \\
\hline 7 & 357,54 & 3,133 & 0,006 \\
\hline
\end{tabular}

Tabela 2: Resultados da simulação e cálculos para a rede quadrada de resistores em função do número de camadas.

\begin{tabular}{lccc}
\hline N. de camadas & $\mathrm{R}_{\text {eq }}(\mathrm{k} \Omega)$ & Cálculo de $\pi$ & $\Delta \pi$ \\
\hline 1 & 400 & 2,800 & - \\
\hline 2 & 374 & 2,995 & 0,195 \\
\hline 3 & 366 & 3,060 & 0,065 \\
\hline 4 & 363 & 3,085 & 0,025 \\
\hline 5 & 361 & 3,102 & 0,017 \\
\hline 6 & 360 & 3,111 & 0,009 \\
\hline 7 & 359 & 3,120 & 0,009 \\
\hline
\end{tabular}

valor da aproximação de $\pi$ com o acréscimo de cada camada.

A figura 4 apresenta um gráfico do valor calculado de $\pi$ experimental e simulado em função do número de camadas da rede quadrada de resistores. É possível ver no gráfico também uma linha tracejada azul que serve de referência para o valor de $\pi$. Vemos claramente que a medida que aumentamos o número de camadas o valor obtido para o número $\pi$ se aproxima do valor 3,1415. . .

Podemos observar na figura 4 que a convergência para o valor real $\pi$ é lenta com o acréscimo de mais camadas Isto também é indicado pelo valor de $\Delta \pi$ apresentado nas tabelas 1 e 2 Com o acréscimo de cada camada o valor de $\Delta \pi$ possui valor cada vez menor. Como a cons-

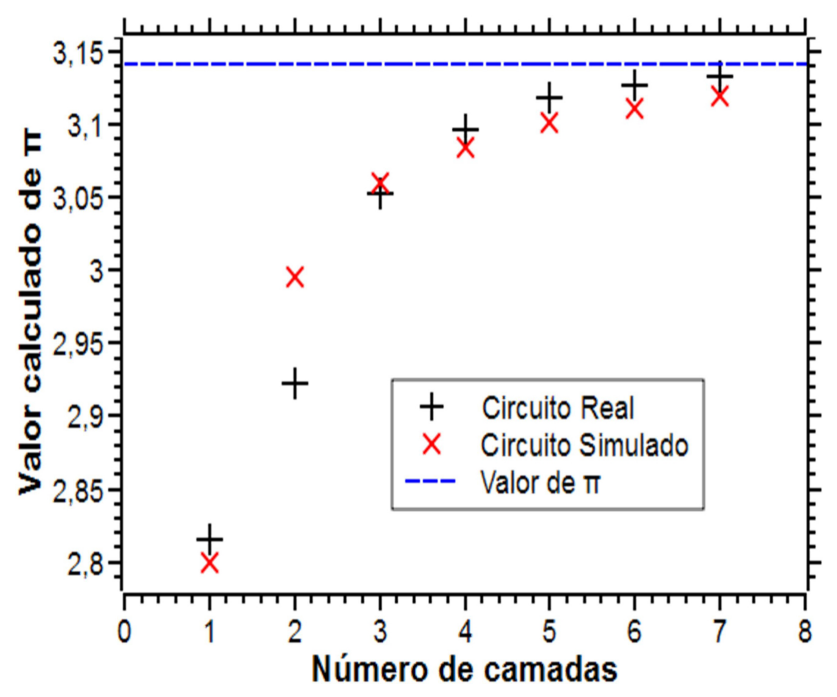

Figura 4: Gráfico de valor calculado para $\pi$ em função do número de camadas. Em azul (tracejado) temos apresentado o valor conhecido de $\pi$ como referência. trução de uma nova camada representa o acréscimo de uma quantidade muito grande de resistores e um incremento pequeno no valor obtido para $\pi$ foi decidido parar na sétima camada. Esse mesmo problema foi discutido por Denardo [5]. Em seu trabalho ele estudou teórica e experimentalmente a rede quadrada finita comparando o resultado encontrado pelos dois métodos. No caso ele construiu uma rede com 6 camadas e encontrou um valor para a razão da resistência equivalente pela resistência dos resistores igual a $R_{\text {eq }} / R=0,643$. Este resultado leva a um valor de $\pi$ igual a 3,110 , bem parecido com os valores que encontramos com 6 camadas construídas.

Finalmente, para realizar de forma viável e barata um experimento como este em sala de aula, sugerimos a mesma execução do experimento com outros materiais. Resistores e soldas são substituídos por traços feitos com um lápis de grafite puro (encontrado em lojas especializadas em material artístico). O grafite não é um bom condutor e as linhas deixadas no papel possuem certa resistência que pode ser calculada facilmente. Neste caso o experimento é feito desenhando, com a ajuda de uma régua, linhas que se cruzam distanciadas igualmente uma das outras, no entanto, todas as nossas tentativas de produzir linhas com a mesma resistividade, igualmente espaçadas, como também, possuírem a mesma espessura, não deram certo. Os resultados que obtivemos não foram satisfatórios devido ao fato de que cada segmento de linha possui uma resistência muito diferente da outra, gerando uma enorme flutuação nos resultados. Para sanar a dificuldade do espaçamento e de espessura com as linhas, pode ser substituído o grafite, por uma caneta de tinta condutora e assim, desenhando as linhas da mesma forma dita anteriormente [6].

\section{Conclusões}

Neste trabalho construímos uma rede quadrada finita de resistores e ao longo de cada etapa da construção medimos a resistência equivalente da diagonal do quadrado central. Esse processo foi feito tanto experimentalmente como através de simulação. Com a resistência equivalente calculamos o valor de $\pi$ com base no resultado esperado para uma rede infinita de resistores, assim, nossos resultados para sete camadas de resistores foram bastante satisfatórios. Outra alternativa para a construção da malha infinita é o uso de grafite, no entanto, demanda que seja desenhado linhas uniformes, pois, a espessura das linhas gera uma grande flutuação nos resultados experimentais. Como alternativa para o grafite seria caneta de tinta condutora, pois, com ela é possível deixar as linhas bem mais uniformes.

\section{Agradecimentos}

Os autores gostariam de agradecer à FUNDECT e ao CNPq pelo apoio. 


\section{Referências}

[1] B. Vincenzo e R. Watanabe, Revista do Professor de Matemática 19, 1 (1991).

[2] E.L. Lima, Revista do Professor de Matemática 6, 18 (1985).

[3] G. Venezian, American Journal of Physics 62, 1000 (1994).

[4] 123D circuits, disponível em https://123d.circuits. io acessado em $15 / 01 / 2017$.

[5] B. Denardo, J. Earwood and V. Sazonova, American Journal of Physics 67, 981 (1999).

[6] Instructables, disponível em http://www instructables.com/id/Estimate-pi-using-

conductive-paint/?ALLSTEPS, acessado em $20 / 01 / 2017$. 